\title{
Single molecule fluorescence microscopy investigations on heterogeneity of translational diffusion in thin polymer films
}

\author{
Bente M. I. Flier, ${ }^{a}$ Moritz Baier, ${ }^{a}$ Johannes Huber, ${ }^{a}$ Klaus Müllen, ${ }^{b}$ \\ Stefan Mecking, ${ }^{a}$ Andreas Zumbusch ${ }^{a}$ and Dominik Wöll ${ }^{* a c}$
}

Translational diffusion of single perylene diimide molecules in $25 \mathrm{~nm}$ thin polymer films was investigated by single molecule widefield fluorescence microscopy. Spatial heterogeneities in single molecule motion were detected and analyzed by a new, quantitative method which draws a comparison of log-Gaussian fits of experimentally determined diffusion coefficient-distributions and diffusion coefficient-distributions from Monte Carlo random walk simulations.

Heterogeneities could be observed close to the glass transition temperature, but disappear at $c a .1 .1 \times T_{\mathrm{g}}$. At higher temperatures, heterogeneities do not exist or they average out on the time and length scales of observation. The observed heterogeneities also explain why the dependency of diffusion coefficients on temperature does not follow Vogel-Fulcher-Tammann behavior.

\section{Introduction}

Despite the millennium-long experience of glass production and processing, neither experimental nor theoretical scientists have been able to elucidate all facets of the glass transition. ${ }^{1-3}$ To a significant extent, it has remained a mystery. It has been stated to be likely the "deepest and most interesting unsolved problem in solid state theory." The glass transition is a ubiquitous phenomenon in many important materials such as silica glass, amorphous metals, colloids, ceramics, supercooled liquids ${ }^{5}$ and polymers. ${ }^{6}$ It can even account for the mechanical properties of animal cells and tissues. ${ }^{7}$ When cooled down fast enough to avoid crystallization, materials exhibit a phase transition from a disordered liquid to a metastable disordered glassy state which behaves mechanically like a solid. The conversion between these states is known as the glass transition. The respective glass transition temperature $T_{\mathrm{g}}$ can be defined in several ways, the most common being the point where a viscosity of $10^{12} \mathrm{~Pa}$ s is reached. However, the cooling rate also determines when the glass transition occurs. Previous investigations have shown that the glass transition is governed by kinetics rather than thermodynamics, i.e. the system is frozen before it can reach its thermodynamically preferred state. ${ }^{8}$ However, it is still poorly understood how molecular dynamics are related to the glass transition.'

\footnotetext{
"Fachbereich Chemie, Universität Konstanz, Universitätsstrasse 10 78464 Konstanz, Germany. E-mail: dominik.woell@uni-konstanz.de Tel: +497531882024

${ }^{b}$ Max-Planck-Institute for Polymer Research, Ackermannweg 10 55128 Mainz, Germany

${ }^{c}$ Zukunftskolleg, Universität Konstanz, Universitätsstrasse 10, 78464 Konstanz, Germany
}

In glass-forming polymers, different relaxation processes can be observed. ${ }^{6}$ The most prominent of these processes are the so-called $\alpha$ - and $\beta$-relaxation, two distinguishable processes which merge above a certain temperature. $\beta$-relaxation refers to local motions of polymer segments or the ends of polymer chains and typically shows an Arrhenius type, exponential temperature dependence. In contrast, $\alpha$-relaxation describes correlated motion of entire regions of the polymer. These correlated motions ${ }^{9}$ appear near $T_{\mathrm{g}}$ when, due to the low free volume within the polymer, the motions of polymer molecules or polymer fragments are no longer free, but coupled to each other in so-called cooperatively rearranging regions with typical sizes of $1-4 \mathrm{~nm}$ (see ref. 10 and references therein). The temperature dependence of the $\alpha$-relaxation can normally be expressed using the so-called Vogel-Fulcher-Tammann (VFT) equation. With decreasing temperature, the size of these cooperative regions becomes larger. One of the main questions concerning $\alpha$-relaxation is whether it is of homogeneous or heterogeneous origin. "In the case of homogeneous relaxation, similar diffusion coefficients are expected for different molecules in the polymer whereas in the heterogeneous case, a distribution of diffusion coefficients can be assumed. ${ }^{12,13}$

Experimentally, diffusion in polymers ${ }^{14}$ has been probed using many different techniques such as pulsed-gradient NMR which allows for detection of small probes ${ }^{15-17}$ and deuterated polymer chains, ${ }^{18}$ forced Rayleigh scattering, ${ }^{19,20}$ dynamic light scattering, ${ }^{21}$ fluorescence recovery after photobleaching (FRAP), ${ }^{22}$ fluorescence correlation spectroscopy (FCS) to determine self-diffusion and cooperative diffusion, ${ }^{17,23}$ dielectric spectroscopy, ${ }^{24}$ Taylor dispersion and phosphorescence quenching, ${ }^{25}$ and Förster resonance energy transfer (FRET) to investigate polymer interdiffusion. ${ }^{26}$ 
All these methods average over many molecules and do not directly elucidate diffusional heterogeneities. However, ways have been found to analyze these. They are often quantified by the exponent $\beta$ of a stretched exponential function (also called Kohlrausch-Williams-Watts function), which in the homogeneous case is 1 (single exponential function) and for heterogeneous systems becomes less than 1. Also, a determination of the distribution of diffusion coefficients in dynamic light scattering experiments is possible by Tikhonov regularization. ${ }^{27}$ The most direct insight is obtained from the observation of the motion of single particles, so-called microrheology, ${ }^{28}$ which yields the distribution of diffusion coefficients. To date, experiments of this type had to rely on particles with radii in the micrometre range which allowed optical observation. Particles of this size, however, cannot probe relaxation processes which occur on much smaller length scales.

Within the last decades, optical microscopy methods have improved considerably enabling the observation of single fluorescent molecules. ${ }^{29-32}$ This has opened new possibilities for the investigation of dynamics in polymers and supercooled liquids at temperatures around the glass transition. Fluorescence lifetime measurements have been used to determine the local free volume around single probes. ${ }^{33.34}$ Rotational motion including spatial and temporal heterogeneities has been detected using single molecule linear dichroism, ${ }^{34-37}$ and defocused widefield imaging with free fluorescent probes ${ }^{38}$ and probes covalently attached to the end of polymer chains. ${ }^{39}$ Schob et al. $^{35}$ have compared single molecule translational motion with rotational motion in order to study the rotationtranslation paradox..$^{40-43}$

Here, we report on temperature dependent single molecule measurements in $25 \mathrm{~nm}$ thin polystyrene films. We present a new approach to detect and analyze spatial heterogeneities based on a comparison between experiment and simulation, and show that heterogeneity increases when approaching the glass transition temperature $T_{\mathrm{g}}$ from high temperatures.

\section{Experimental methods and analysis}

\section{Setup}

A $561 \mathrm{~nm}$ solid state laser (Cobolt Jive, $75 \mathrm{~mW}$ ) was used to excite the dye molecules. The laser power was adjusted with neutral density filters. To obtain homogeneous illumination, the laser was coupled into a multi-mode fibre (NA $0.22 \pm 0.02$, Optronis) which was shaken to destroy coherence and suppress interference effects detrimental to homogeneous sample illumination. The end of the fibre was imaged onto the sample to a spot of $50 \mu \mathrm{m}$ diameter yielding an intensity of about $1-4 \mathrm{~kW} \mathrm{~cm}^{-2}$. The fuorescence was collected using a $63 \times 10.75 \mathrm{NA}$ air objective (Leica HI Plan), separated from the laser light with a dichroic mirror (AHF, z561 660 rpc) and a longpass filter (AHF, RazorEdge LP 568RU), further magnified by two photo-objectives (Nikkor $28 \mathrm{~mm}$ and $86 \mathrm{~mm}$ ) and imaged onto an EMCCD camera (Andor iXON). The total magnification resulted in an image size of $86 \mathrm{~nm}$ per pixel of the CCD camera. Samples were resistively heated using a custom built device. Temperatures were controlled in a feedback fashion using an IR thermometer.

\section{Sample preparation}

Sample substrates were cleaned using ultrasonication in isopropanol (spectroscopic grade, Acros organics) and in an oxygen plasma oven (Plasmacleaner Femto, Diener Electronics). Polystyrene of molecular weight $M_{\mathrm{W}}=3000 \mathrm{~g} \mathrm{~mol}^{-1}$ (Polymer Source, Inc.) and a perylene diimide derivative ( $N, N^{\prime}$-bis(2,6-diisopropylphenyl)-1,6,7,12-tetra[4-(1,1,3,3-tetramethylbutyl)phenoxy]-perylene-3,4:9,10-tetracarboxylic diimide $)^{44}$ were dissolved in toluene $(99+\%$, spectroscopic grade, Acros organics) to obtain a solution of $0.5 \mathrm{wt} \%$. This polymer concentration was chosen in order to produce films of $25 \mathrm{~nm}$ thickness. The solution was spin coated at $2000 \mathrm{rpm}$. Subsequently the samples were dried under vacuum for $24 \mathrm{~h}$ to remove any remaining toluene from the films. ${ }^{45}$ To investigate differences in single molecule data occurring due to stresses from spin coating, some samples were heated up to $80^{\circ} \mathrm{C}$ under vacuum during evaporation of the solvent for $24 \mathrm{~h}$ (referred to as relaxed samples), while others were cooled down to $-30{ }^{\circ} \mathrm{C}$ under vacuum to avoid relaxation (referred to as non-relaxed samples). The film thickness of the samples was measured after single molecule measurements by scratching the polymer film and measuring the depth of the scratch by AFM.

\section{SM tracking}

Different ways for single molecule tracking have been described in the literature. ${ }^{46-53}$ Based on some of these methods we used a home-made procedure which will be described in the following section. After filtering the images, the positions of single molecules were determined by calculating the center of mass of the corresponding spot. For our systems, this gave similar results and accuracies as $2 \mathrm{D}$-Gaussian fitting of the same spots, but reduced the calculation times considerably. Tracking was done by connecting the positions of corresponding spots with each other from frame to frame. A semi-automatic algorithm was chosen which, if possible, connected the positions automatically. Otherwise, user input was required to find an appropriate spot when none or several signals were present in the vicinity of the previous position, to continue with tracking after blinking, or to determine irreversible photobleaching. This procedure avoids tracking errors which we found in fully automatic tracking routines and which can significantly influence the results. Diffusion coefficients of single molecules were calculated by a mean square displacement analysis according to Kues et $\mathrm{ll}^{48}$ Step length distributions of all pairs of points were plotted for different lag times from the CCD-integration time $t_{\text {int }}$ up to $10 \times t_{\text {int }}$. These step length distributions were fitted by an expression for random diffusion in two dimensions: $:^{54}$

$$
P\left(r, t_{\text {lag }}\right)=\frac{2 \pi r}{4 \pi D t_{\text {lag }}} \exp \left(-\frac{r^{2}}{4 D t_{\text {lag }}}\right)
$$

The value $4 D t_{\text {lag }}$ can be obtained by fitting this function to the step length distributions for fixed $t$. Repeating this for all lag time intervals $t_{\text {int }}, 2 \times t_{\text {int }}, 3 \times t_{\text {int }}$ etc. up to $10 \times t_{\text {int }}$ results in a graph of $4 D t_{\text {lag }} v s . t_{\text {lag }}$ with the slope $4 D$. The value for $D$ was 

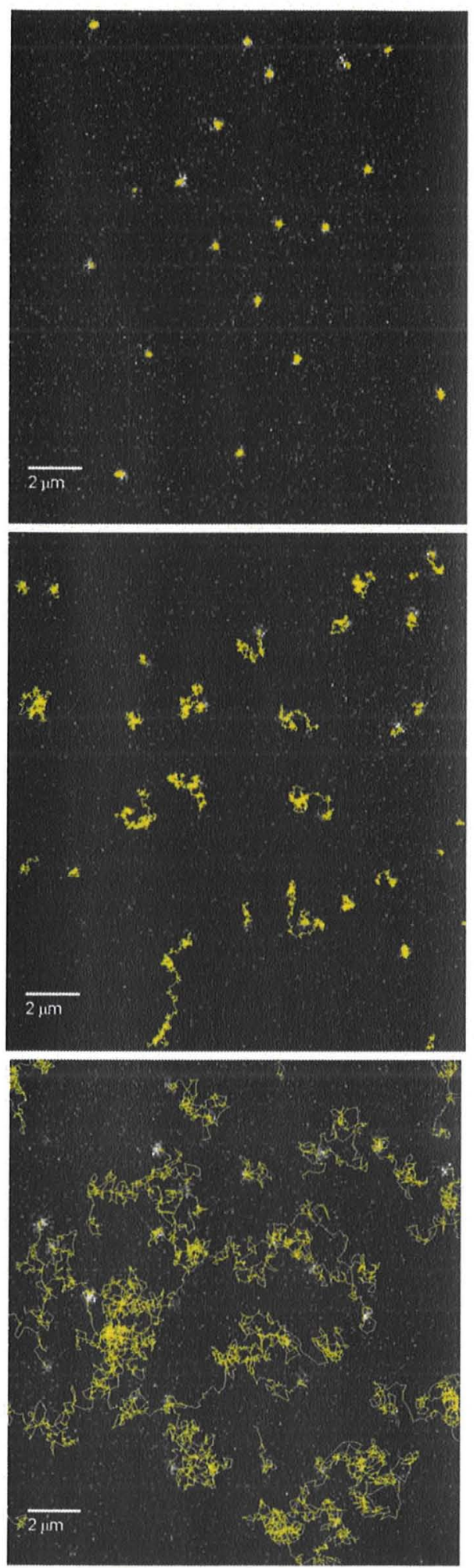

Fig. 1 Typical images of single molecules in a non-relaxed, thin PS3000 film including single molecule tracks with up to 500 steps at an integration time of $300 \mathrm{~ms}$ for $T=358 \mathrm{~K}, 378 \mathrm{~K}$ and $398 \mathrm{~K}$.

taken as the diffusion coefficient of the single molecule under consideration.

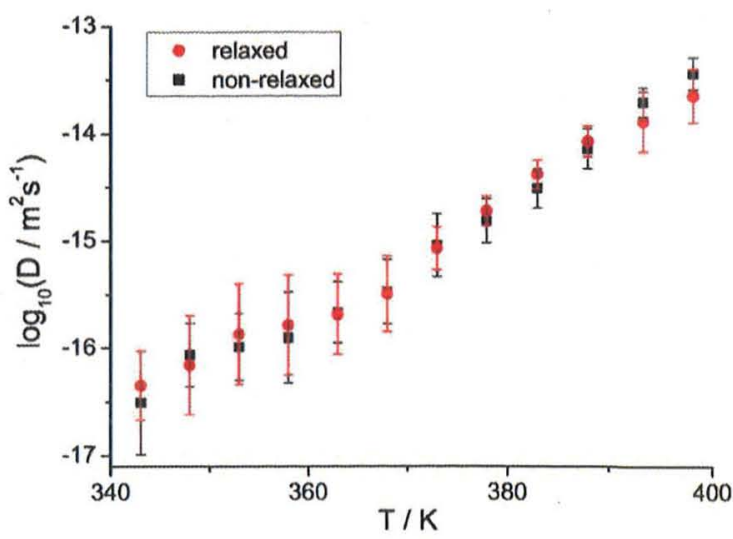

Fig. 2 Average diffusion coefficients of the mobile fraction and widths taken from Gaussian fit of single molecule distributions of diffusion coefficients.

\section{Results}

Temperature dependent dynamics of single molecules

The dynamics of single perylene diimide (PDI) dye molecules embedded in a $25 \mathrm{~nm}$ thin polystyrene film $\left(M_{\mathrm{w}} 3000 \mathrm{~g} \mathrm{~mol}^{-1}\right.$, polydispersity 1.07 , bulk $T_{\mathrm{g}} 342 \mathrm{~K}$ ) was detected by widefield fluorescence microscopy. Measurements were performed at temperatures ranging from $298 \mathrm{~K}$ to $398 \mathrm{~K}$ in steps of $5 \mathrm{~K}$. Connecting the positions of molecules in each movie resulted in tracks such as shown in Fig. 1 for three different temperatures. Mobility of the molecules was determined by analysis of steplength distributions (see Experimental section) in two different ways: (a) the step lengths of all (mobile) molecules were combined and analyzed together or (b) diffusion coefficients of single molecules were determined by step length analysis, their distributions plotted and fitted by log-Gaussian functions with their maximum defined as the average diffusion coefficient.

The average diffusion coefficients and the standard deviation of the Gaussian fits for relaxed and non-relaxed polymer films are plotted in Fig. 2. There is no significant difference in the average $D$ of relaxed and non-relaxed polymer films. Both increase constantly with increasing temperature in a non-trivial dependence on $T$. Assuming Stokes-Einsteinbehavior, the diffusion coefficient $D$ is inversely proportional to the viscosity $\eta$ which, in a bulk polymer, is expected to depend on $T$ according to the Vogel-Fulcher-Tammann law ${ }^{55}$ which is expected to be valid for the temperature range investigated in our study. Thus, if as expected, the free dye molecules probe the structural $\alpha$-relaxation, $D$ is expected to obey the relationship

$$
D=D_{0} \exp \left(-\frac{B}{R_{0}\left(T-T_{0}\right)}\right)
$$

with the diffusion coefficient $D_{0}$ in the limit of high $T$, an activation energy equivalent parameter $B$, the ideal gas constant $R_{0}$, and the Vogel temperature $T_{0}$. Such a dependence can, however, not be obtained from the data in Fig. 2. An explanation for this phenomenon is heterogeneity within the thin polymer film which causes different dynamics and thus 


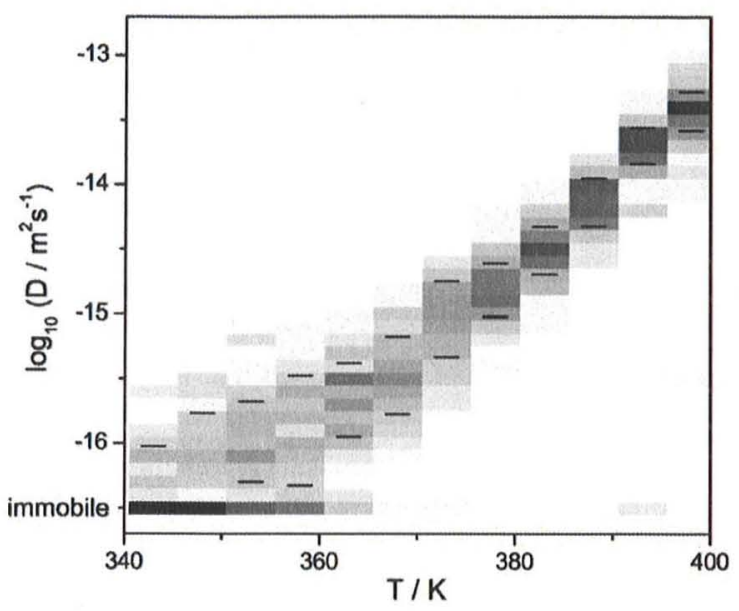

Fig. 3 Contour plot of the distributions of single molecule diffusion coefficients for a $25 \mathrm{~nm}$ thin, non-relaxed PS3000 film. The standard deviation of log-Gaussian fits at each temperature is represented as black lines.

different Vogel-Fulcher-Tammann parameters at different positions in the film.

The single molecule approach allows us not only to determine average values with their standard deviation (see Fig. 2) but also to visualize distributions of diffusion coefficients as shown in the contour plot in Fig. 3. Here, it becomes obvious that the distribution becomes significantly broader when approaching the bulk $T_{\mathrm{g}}$. We assured that the broadening of the distribution is not due to the positioning accuracy using reference measurement of single PDI molecules immobilized in polystyrene far below its $T_{\mathrm{g}}$. Furthermore, at $T<370 \mathrm{~K}$, an immobile fraction of molecules appears which both by analysis via corresponding diffusion coefficients $\left(D<10^{-16.5} \mathrm{~m}^{2} \mathrm{~s}^{-1}\right)$ and by eye can clearly be distinguished from the mobile molecules. Below the bulk $T_{\mathrm{g}}$, no single molecule mobility could be observed by the techniques used.

\section{Heterogeneities in single molecule diffusion coefficients}

Heterogeneities can be divided into spatial and temporal heterogeneities. ${ }^{3}$ Spatially heterogeneous motion is observed when molecules in different regions show different velocities whereas the motion of a single molecule can be dynamically heterogeneous when, for example due to changes in its surrounding, it significantly changes velocity over time. Spatial and temporal heterogeneities in single molecule rotation could be observed by defocused widefield-imaging. ${ }^{38}$ In our measurements of translational diffusion, we were able to detect spatial heterogeneities, but the velocity remained constant for most molecules within our observation time. Thus for most molecules no significant dynamic heterogeneities could be observed.

In order to analyze the data, we developed a new approach to investigate spatial heterogeneity in single molecule translational diffusion. It consists of a comparison between the widths of measured and simulated distributions of single molecule diffusion coefficients. The experimental data were determined by mean square displacement analysis according to Kues et $a l .^{48}$ (see Experimental part). Molecules with a

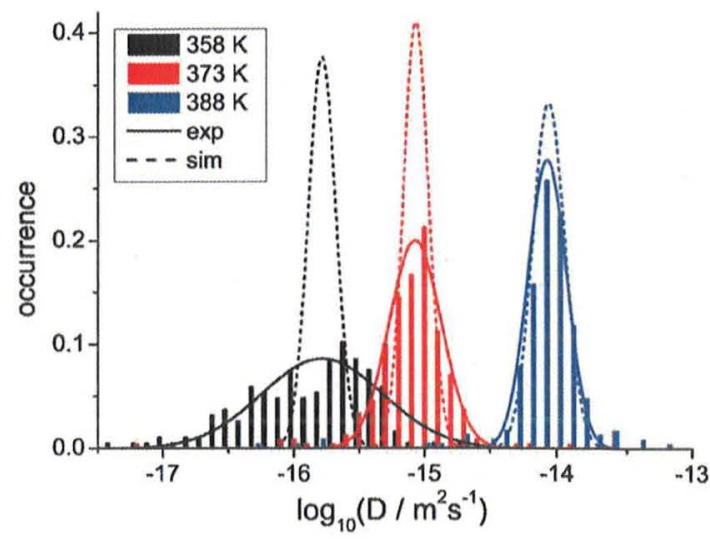

Fig. 4 Distributions of diffusion cocfficients $D$ of a $25 \mathrm{~nm}$ thin, relaxed PS3000 film at three different temperatures. The experimental distributions are fitted with a log-normal distribution (eqn (3)) and compared to simulated data (see text).

track length shorter than 30 steps were not included because simulations showed that reliable diffusion coefficients can only be obtained for tracks large enough to allow for appropriate statistics. Histograms of the logarithm of diffusion coefficients were fitted by a Gaussian function

$$
y=\frac{A}{\sigma \sqrt{2 \pi}} \exp \left(-\frac{\left(x-x_{0}\right)^{2}}{2 \sigma^{2}}\right)
$$

with the standard deviation $\sigma$, the mean value $x_{0}$ and a normalization factor $A$ (in our case set to 0.1 ) accounting for the binning width in $\log D$. The widths $\sigma_{\text {measured }}$ of these distributions, however, are not yet an appropriate measure for heterogeneity in the system. As pointed out by Saxton, ${ }^{56}$ distributions become broader for smaller track lengths. Thus, even molecules diffusing with the same $D$ will result in rather broad distributions after analysis, due to the finite track lengths. In order to take this effect into account, we performed Monte Carlo random walk simulations of trajectories with the average $D$ and the step length distribution taken from the corresponding measurement. The obtained $\log D$-distributions were analyzed with the same method as the experimental

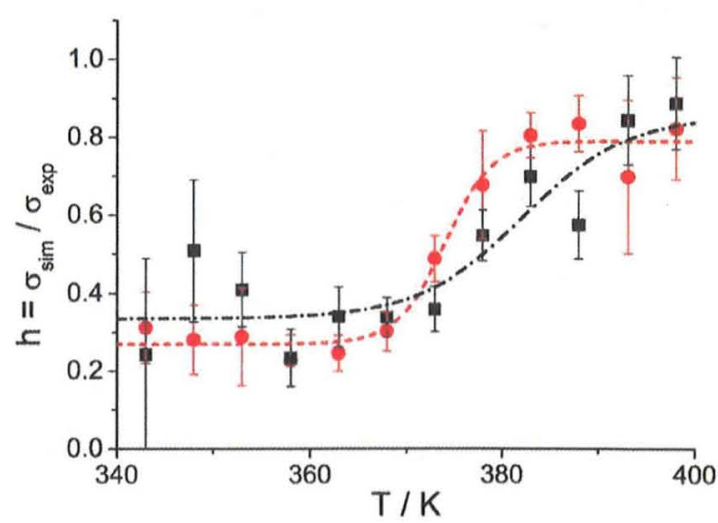

Fig. 5 Heterogeneity of diffusion coefficients in relaxed and nonrelaxed PS3000 films at different temperature including guides to the eyes. 
distributions using eqn (3). This procedure resulted in distributions with a width $\sigma_{\text {simulated }}$ which contains the contribution of the $\log D$-distribution assuming random walk behavior and the broadening due to the finite trajectory length. A comparison between these data and the experimentally determined $\log D$-distributions then gives direct insight into the heterogeneities of the sample.

Fig. 4 shows three examples of distributions with different $D$-range (average $D=1.64 \times 10^{-16} \mathrm{~m}^{2} \mathrm{~s}^{-1}, 8.51 \times 10^{-16} \mathrm{~m}^{2} \mathrm{~s}^{-1}$ and $\left.8.49 \times 10^{-15} \mathrm{~m}^{2} \mathrm{~s}^{-1}\right)$ and different widths. Gaussian fits of the $\log D$-distributions are shown for the experimental (solid lines) and simulated data (dashed lines). The corresponding widths of these Gaussian fits are $\sigma_{\text {measured }}$ and $\sigma_{\text {simulated, }}$, respectively. The ratio $h=\sigma_{\text {simulated }} / \sigma_{\text {measured }}$ is close to 1 for homogeneous systems, where molecules diffuse with similar $D$. If $D$ for single molecules are distributed, the width of the measured distribution becomes broader and thus the ratio $h$ decreases. Thus, small $h$-values characterize heterogeneous systems. A plot of these $h$-values versus temperature for the relaxed and non-relaxed films is shown in Fig. 5.

\section{Discussion}

Our results show that significant heterogeneities of the motion of probes in thin polymer films appear when approaching $T_{\mathrm{g}}$ from high temperatures. With some exceptions, heterogeneities could only be observed in the diffusion between molecules (spatial heterogeneities), but not within the diffusion of single molecules (dynamic heterogeneities). This means that significant dynamic heterogeneities either do not exist or they are too slow or too fast to be detected by our translation measurement. Significant spatial heterogeneities could be observed between $340 \mathrm{~K}$ and $380 \mathrm{~K}$, i.e. between bulk $T_{\mathrm{g}}$ and $1.1 \times T_{\mathrm{g}}$, in the $25 \mathrm{~nm}$ thin film (see Fig. 5). Above $380 \mathrm{~K}$, translational motion was homogeneous. This is in agreement with observations by Schob et al., who found no heterogeneities in the translational motion of single rhodamine $6 \mathrm{G}$ molecules above $1.2 \times T_{\mathrm{g}}$ in an $80 \mathrm{~nm}$ thick PMA $\left(40 \mathrm{~kg} \mathrm{~mol}^{-1}\right)$ film. ${ }^{35}$ At higher temperatures, heterogeneities do not exist or they average out on our time- and length scales of observation.

The observed heterogeneities also explain why the dependency of diffusion coefficients on temperature does not show VogelFulcher-Tammann (VFT) behavior as it would be expected in a bulk polymer. ${ }^{19}$ The VFT parameters for molecules in different surroundings are variable and thus the observed change of average diffusion coefficient with temperature is less steep.

In order to elucidate effects on sample preparation which affect translational diffusion, we compared translational motion in non-relaxed and relaxed samples. No significant differences can be observed for the average values of $D$ in Fig. 2. However, the transition from heterogeneous to homogeneous motion, expressed by a change in the corresponding $h$-values as shown in Fig. 5 , occurs earlier and more rapidly in the relaxed films. We assume that in the non-relaxed films the stress starts to release gradually and thus heterogeneities take longer to disappear compared to the film already relaxed before. In the relaxed films most stress has already been released by heating to $80{ }^{\circ} \mathrm{C}$ for $24 \mathrm{~h}$ before the measurements. In addition, the heterogeneity data of the relaxed films scatter significantly less compared to the ones in the non-relaxed films. This shows that the pre-heating step makes the films more uniform than they would be directly after spin-coating.

\section{Conclusions}

With this paper we demonstrated the power of single molecule microscopy to investigate dynamics within thin polymer films. We measured and analysed the temperature-dependent translational diffusion of single dye molecules in $25 \mathrm{~nm}$ thin polystyrene films. The single molecule approach allowed us to observe changes in heterogeneity when approaching $T_{g}$ which were further analyzed using a new approach for the quantification of spatial heterogeneities. This approach is based on a comparison of log-Gaussian fits of our experimentally determined diffusion coefficient-distributions and diffusion coefficient-distributions from Monte Carlo random walk simulations with the same step length distributions.

Heterogeneities could be observed close to the glass transition temperature, but they disappear at temperatures above $c a$. $1.1 \times T_{\mathrm{g}}$. This is consistent with observations of Schob et al., ${ }^{35}$ who did not observe heterogeneities in translational motion above $1.2 \times T_{\mathrm{g}}$. The heterogeneities in the sample explain why the dependency of diffusion coefficients on temperature does not show Vogel-Fulcher-Tammann behavior.

\section{Acknowledgements}

Financial support of the Zukunftskolleg of the University of Konstanz and the Center for Mesoscopic Structures within the Exzellenzinitiative is gratefully acknowledged. B.M.I.F and M.C.B. thank the Fonds der Chemische Industrie for Ph.D. scholarships.

\section{Notes and references}

1 C. A. Angell, Science, 1995, 267, 1924-1935.

2 R. Schilling, in Collective Dynamics of Nonlinear and Disordered Systems, ed. G. Radons, W. Just and P. Häussler, Springer, 2003. 3 M. D. Ediger, C. A. Angell and S. R. Nagel, J. Phys. Chem., 1996, $100,13200-13212$.

4 P. W. Anderson, Science, 1995, 267, 1609-1618.

5 P. G. Debenedetti and F. H. Stillinger, Nature, 2001, 410, 259-267.

6 R. Zorn, J. Phys.: Condens. Matter, 2003, 15, R1025-R 1046.

7 C. Semmrich, T. Storz, J. Glaser, R. Merkel, A. R. Bausch and K. Kroy, Proc. Natl. Acad. Sci. U. S. A., 2007, 104, 20199-20203.

8 B. Jérôme and J. Commandeur, Nature, 1997, 386, 589-592.

9 C. Bennemann, C. Donati, J. Baschnagel and S. C. Glotzer, Nature, 1999, 399, 246-249.

10 C. J. Ellison, M. K. Mundra and J. M. Torkelson, Macromolecules, 2005, 38, 1767-1778.

11 R. Richert, J. Phys.: Condens. Matter, 2002, 14, R703-R738.

12 H. Sillescu, J. Non-Cryst. Solids, 1999, 243, 81-108.

13 M. D. Ediger, Annu. Rev. Phys. Chem., 2000, 51, 99-128.

14 Diffusion in Polymers, ed. P. Neogi, Marcel Dekker, Inc., New York, Basel, Hong Kong, 1996.

15 E. von Meerwall and R. D. Ferguson, J. Appl. Polym. Sci., 1979, 23, 3657-3669.

16 M. P. Tonge and R. G. Gilbert, Polymer, 2001, 42, 1393-1405. 
17 G. Modesti, B. Zimmermann, M. Börsch, A. Herrmann and K. Saalwächter, Macromolecules, 2009, 42, 4681-4689.

18 K. Kamiguchi, S. Kuroki, M. Satoh and I. Ando, Macromolecules, $2008,41,1318-1322$.

19 D. Ehlich and H. Sillescu, Macromolecules, 1990, 23, 1600-1610.

20 W. J. Huang, T. S. Frick, M. R. Landry, J. A. Lee, T. P. Lodge and M. Tirrell, AIChE J., 1987, 33, 573-582.

21 J. Won, C. Onyenemezu, W. G. Miller and T. P. Lodge, Macromolecules, 1994, 27, 7389-7396.

22 S. Seiffert and W. Oppermann, Polymer, 2008, 49, 4115-4126

23 U. Zettl, S. T. Hoffmann, F. Koberling, G. Krausch, J. Enderlein, L. Harnau and M. Ballauff, Macromolecules, 2009, 42, 9537-9547.

24 O. van den Berg, W. G. F. Sengers, W. F. Jager, S. J. Picken and M. Wübbenhorst, Macromolecules, 2004, 37, 2460-2470.

25 M. B. Wisnudel and J. M. Torkelson, Macromolecules, 1996, 29, 6193 6207.

26 Y. Q. Liu, J. C. Haley, K. Deng, W. Lau and M. A. Winnik, Macromolecules, 2007, 40, 6422-6431.

27 A. N. Tikhonov and V. Arsenin, Solution of $11 \mathrm{l}$-posed Problems, Wiley, New York, 1977.

28 T. A. Waigh, Rep. Prog. Phys., 2005, 68, 685-742.

29 J. M. Lupton, Adv. Mater., 2010, 22, 1689-1721.

30 F. Kulzer, T. Xia and M. Orrit, Angew. Chem., Int. Ed., 2010, 49, $854-866$.

31 D. Wöll, E. Braeken, A. Deres, F. De Schryver, H. Uji-i and J. Hofkens, Chem. Soc. Rev., 2009, 38, 313-328.

32 P. Tinnefeld and M. Sauer, Angew. Chem., Int. Ed., 2005, 44, 2642-2671.

33 R. A. L. Vallée, M. Van der Auweraer, F. C. De Schryver, D. Beljonne and M. Orrit, ChemPhysChem, 2005, 6, 81-91.

34 E. Braeken, G. De Cremer, P. Marsal, G. Pepe, K. Müllen and R. A. L. Vallée, J. Am. Chem. Soc, 2009, 131, 12201-12210.

35 A. Schob, F. Cichos, J. Schuster and C. von Borczyskowski, Eur. Polym. J., 2004, 40, 1019-1026.

36 R. Zondervan, F. Kulzer, G. C. G. Berkhout and M. Orrit, Proc. Natl. Acad. Sci. U.S. A., 2007, 104, 12628-12633.

37 S. A. Mackowiak, T. K. Herman and L. J. Kaufman, J. Chem. Phys., 2009, 131, 244513.
38 H. Uji-i, S. M. Melnikov, A. Deres, G. Bergamini, F. De Schryver, A. Herrmann, K. Müllen, J. Enderlein and J. Hofkens, Polymer, $2006,47,2511-2518$.

39 Z. L. Zheng, F. Y. Kuang and J. Zhao, Macromolecules, 2010, 43, 3165-3168.

40 F. H. Stillinger and J. A. Hodgdon, Phys. Rev. E: Stat. Phys. Plasmas, Fluids, Relat. Interdiscip. Top., 1996, 53, 2995-2997.

41 D. B. Hall, A. Dhinojwala and J. M. Torkelson, Phys. Rev. Lett., $1997,79,103-106$

42 M. T. Cicerone, F. R. Blackburn and M. D. Ediger, Macromole* cules, 1995, 28, 8224 8232

43 M. T. Cicerone, P. A. Wagner and M. D. Ediger, J. Phys. Chem. B, $1997,101,8727-8734$

44 J. Q. Qu, J. Y. Zhang, A. C. Grimsdale, K. Müllen, F. Jaiser, X. H. Yang and D. Neher, Macromolecules, 2004, 37, 8297-8306

45 X. H. Zhang, K. G. Yager, S. H. Kang, N. J. Fredin, B. Akgun, S. Satija, J. F. Douglas, A. Karim and R. L. Jones, Macromolecules, 2010, 43, 1117-1123.

46 M. K. Cheezum, W. F. Walker and W. H. Guilford, Biophys. J. 2001, 81, 2378-2388.

47 M. J. Saxton, Nat. Methods, 2008, 5, 671-672.

48 T. Kues, R. Peters and U. Kubitscheck, Biophys. J., 2001, 80, $2954-2967$.

49 G. J. Schütz, H. Schindler and T. Schmidt, Biophys. J., 1997, 73, $1073-1080$.

50 A. Schob, M. Pumpa, M. Selmke and F. Cichos, J. Phys. Chem. C, $2010,114,4479-4485$

51 C. Hellriegel, J. Kirstein and C. Bräuchle, New J. Phys., 2005, 7, $1-14$.

52 K. Jaqaman, D. Loerke, M. Mettlen, H. Kuwata, S. Grinstein, S. L. Schmid and G. Danuser, Nat. Methods, 2008, 5, 695-702.

53 I. F. Sbalzarini and P. Koumoutsakos, J. Struct. Biol., 2005, 151, $182-195$

54 C. M. Anderson, G. N. Georgiou, I. E. G. Morrison, G. V. W. Stevenson and R. J. Cherry, J. Cell Sci., 1992, 101, 415-425.

55 M. Rubinstein and R. H. Colby, Polymer Physics, Oxford University Press, Oxford, 2003.

56 M. J. Saxton, Biophys. J., 1997, 72, 1744-1753. 